\title{
Note: Highly sensitive superconducting quantum interference device microsusceptometers operating at high frequencies and very low temperatures inside the mixing chamber of a dilution refrigerator
}

\author{
M. J. Martínez-Pérez, ${ }^{1}$ J. Sesé, ${ }^{2}$ F. Luis, ${ }^{1}$ D. Drung, ${ }^{3}$ and T. Schurig ${ }^{3}$ \\ ${ }^{1}$ Instituto de Ciencia de Materiales de Aragón, CSIC-University of Zaragoza, and Departamento de Fisica de \\ la Materia Condensada, University of Zaragoza, Pedro Cerbuna 12, 50009 Zaragoza, Spain \\ ${ }^{2}$ Instituto de Nanociencia de Aragón, and Departamento de Fisica de la Materia Condensada, University of \\ Zaragoza, Pedro Cerbuna 12, 50009 Zaragoza, Spain \\ ${ }^{3}$ Physikalisch-Technische Bundesanstalt (PTB), Abbestraße 2-12, D-10587 Berlin, Germany
}

(Received 20 July 2009; accepted 8 December 2009; published online 25 January 2010)

\begin{abstract}
We report the experimental results that show the operation of superconducting quantum interference device (SQUID) microsusceptometers immersed in the ${ }^{3} \mathrm{He}-{ }^{4} \mathrm{He}$ mixture inside the mixing chamber of a dilution refrigerator at high frequency $(1 \mathrm{MHz})$ and down to very low temperatures $(13 \mathrm{mK})$. The devices are based on highly sensitive and easy-to-use commercial SQUID sensors. The integrated susceptometers are fabricated by rerouting some connections of the SQUID's input circuit. Examples of measurements on molecular magnets $\mathrm{Mn}_{12}$ and $\mathrm{HoW}_{10}$ are shown.
\end{abstract}

(C) 2010 American Institute of Physics. [doi:10.1063/1.3280169]

The research on the properties of magnetic materials at very low temperatures has been stimulated in the last fifteen years by the observation of fascinating quantum phenomena, such as quantum tunneling of spins, ${ }^{1}$ quantum coherence, ${ }^{2-5}$ and quantum entanglement. ${ }^{6,7}$ Besides providing a direct hindsight on the not yet fully understood quantum-toclassical transition, these phenomena might also find application in the development of solid-state quantum information technologies. ${ }^{8}$ Their study has fuelled the development of new magnetic sensors with improved performances that approach the experimental limits in terms of sensitivity and operation conditions. Experiments need to be performed at very low temperatures, when zero-point quantum fluctuations become dominant over classical thermal fluctuations. A very high spin sensitivity is also desirable to enable detecting the magnetic response of single layers of nanomagnets and eventually of individual ones. In addition, by magnetically diluting the samples, the decoherence caused by spin-spin interactions can be minimized. Another crucial point is the access to broader frequency bandwidths than those offered by commercial magnetometers, since the time scales of quantum dynamics can vary over many decades depending on the spin value, the magnetic anisotropy, and the couplings to the environment. The many recent advances in this field include the development of miniature magnetometers, based on the use of either micro-Hall bars or micro- and nanosuperconducting quantum interference device (SQUID) sensors. ${ }^{9}$ Another application with similar stringent performance is found in the use of metallic magnetic microcalorimeters as radiation detectors. ${ }^{10}$

Our purpose has been to develop an ac magnetic susceptometer that combines high sensitivity and broad frequency bandwidth with operation down to very low temperatures and user friendliness. Commercial SQUID technology and readout electronics (e.g., from Magnicon GbR, Germany) are now available with sensitivities near the quantum limit at $\mathrm{mK}$ temperatures and room temperature electronics adapted for operation with broad bandwidths. ${ }^{11}$ We have taken advan- tage of such optimized devices as the starting point for the fabrication of our susceptometers. It turns out that, via a simple modification of the chip's input wiring, a fully integrated thin-film SQUID susceptometer can be obtained. This modification is carried out by focused ion beam etching and the subsequent in situ deposition of a superconducting material with nanoscale control. ${ }^{12-14}$ The problem of achieving a good thermal contact at very low temperature has been solved by immersing the microsusceptometer inside the mixing chamber of a ${ }^{3} \mathrm{He}-{ }^{4} \mathrm{He}$ dilution refrigerator (Leiden Cyrogenics) adapted to this end; this ensures a good thermalization of the sample down to $\approx 13 \mathrm{mK}$.

All the coils in the chip's layout of the Magnicon/PTB SQUID sensors have a gradiometric design in order to minimize the sensitivity to homogenous magnetic fields. For sensors with high input inductance, a double-transformer scheme is used to couple a low-inductance SQUID to a highinductance input coil (see Fig. 1). The input signal is first coupled to an intermediate loop and the flux is then transferred to the front-end SQUID through a second flux transformer. This SQUID incorporates an additional positive feedback and it is read out with a second-stage array of 16 SQUIDs (flux noise at $T=4.2 \mathrm{~K}$ is $S_{\Phi}^{1 / 2} \approx 800 \mathrm{n}_{0} / \mathrm{Hz}^{1 / 2}$ ). ${ }^{11}$ Sensors with a low input-coil inductance do not use the intermediate loop. The input coil is then directly coupled to the front-end SQUID. We have converted these chips into SQUID-susceptometers ${ }^{13}$ with the diagram shown in Figure 1.

Sensors are mounted on printed circuit board (PCB) holders and installed inside the mixing chamber of a dilution refrigerator (see Fig. 2). This chamber is made of plastic and has 24 electrical connections with vacuum tight feedthroughs. To reduce the effect of electromagnetic interferences, a small $\mathrm{Pb}$ shield covers the SQUID holder inside the mixing chamber and a Cryoperm shield surrounds the whole inner vacuum chamber (IVC) of the refrigerator. In addition, special care has been taken to electrically isolate the cryostat and connecting tubes and to rf-filter all the lines 


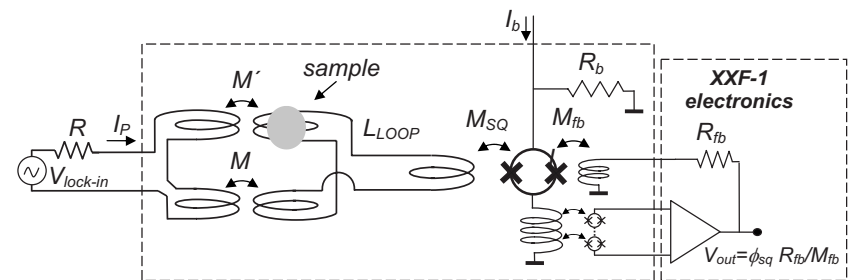

FIG. 1. Schematic diagram of the SQUID susceptometer chip (inside dashed line). Input current $I_{P}$ is controlled with the internal oscillator of the lock-in amplifier $V_{\text {lock-in }}$ and series resistance $R$. $I_{P}$ couples flux to the first order gradiometer of the intermediate loop through mutual inductances $M$ and $M^{\prime}$. These mutual inductances have the same geometry and their values differ only when a magnetic sample is present, in that case $M^{\prime}=M(1+F \chi)$, where $\chi$ is the magnetic susceptibility of the sample and $F$ is a calibration factor. The flux coupled by the signal-SQUID is given by $\phi_{\mathrm{SQ}}=I_{P} M_{\mathrm{SQ}}\left(M^{\prime}\right.$ $-M) / L_{\mathrm{LOOP}}=I_{P} M_{\mathrm{SQ}} F \chi M / L_{\mathrm{LOOP}}$. A second stage 16 -SQUIDs array is used to read-out $\phi_{\mathrm{SQ}}$. Magnicon XXF-1 electronics is used to operate the system in flux-locked loop (FLL) mode. Finally, the in-phase and out-of-phase components of $V_{\text {out }}$ are read-out with the lock-in amplifier.

used for thermometry. A commercial low-thermal conductivity cryocable from Magnicon was used for the wiring from the $\mathrm{mK}$ region to $300 \mathrm{~K}$ at the top of the cryostat were the XXF-1 Magnicon read-out electronics is connected.

The thermalization of the sensor and the sample is ensured by the direct contact with the ${ }^{3} \mathrm{He}-{ }^{4} \mathrm{He}$ mixture of the dilution refrigerator. In this way, the heating of the sample due to the absorption of energy from the ac magnetic field (when the imaginary susceptibility component $\chi^{\prime \prime}$ is not zero) is also prevented since we profit the most from the cooling power of the refrigerator. We have observed that cooling power and base temperature $(13 \mathrm{mK})$ have not been affected. Decreasing the temperature of the SQUID sensors also modifies some of their electronic properties. The critical current of the SQUID array increases a few $10 \%$ below $1 \mathrm{~K}$. However, this change implies no distortion in the $V$ - $\Phi$ curve at very low temperatures. More importantly, the flux noise spectrum, measured at different temperatures (see Fig. 3), shows that the white noise decreases with the square root of temperature until it saturates, below approximately $300 \mathrm{mK}$, probably
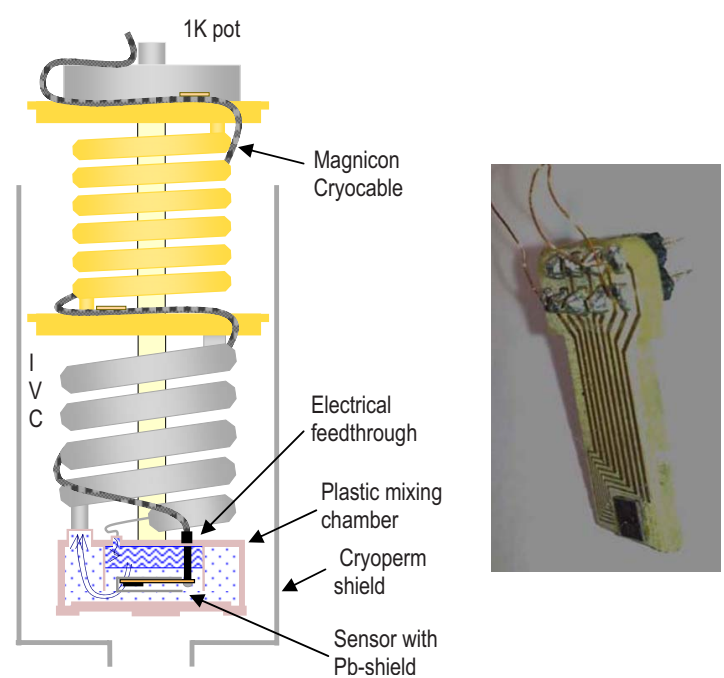

FIG. 2. (Color online) (Left) Representation of the lower part of the dilution refrigerator insert. The sensor lies inside the mixing chamber immersed in the ${ }^{4} \mathrm{He}$ rich phase, with the PCB holder plugged into electrical feedthroughs connected to the cryocable in the IVC. (Right) photograph of SQUIDsusceptometer chip mounted on PCB holder, it can be easily manipulated for changing sample. Chip size is $3 \times 3 \mathrm{~mm}^{2}$.

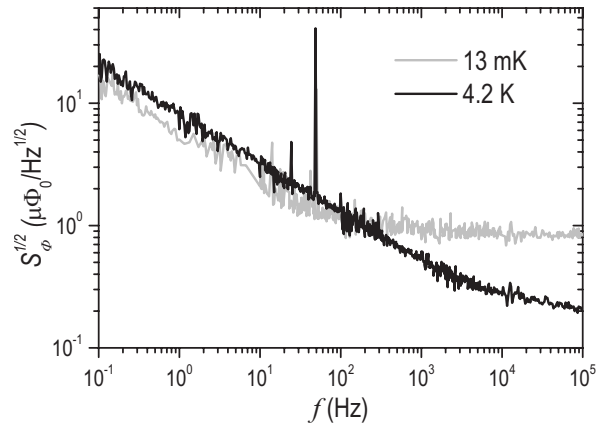

FIG. 3. Flux noise of the sensor measured at $4.2 \mathrm{~K}$ and $13 \mathrm{mK}$ with a HP $3562 \mathrm{~A}$ dynamic signal analyzer (limited to $100 \mathrm{kHz}$ ).

due to hot-electron effects. ${ }^{14}$ At $13 \mathrm{mK}$ and $100 \mathrm{kHz}$, an uncoupled energy sensitivity $S_{\Phi} / 2 L=7 \hbar$ is obtained, where $S_{\Phi}^{1 / 2}=0.20 \mu \Phi_{0} / \mathrm{Hz}^{1 / 2}$ is the magnetic flux noise, $L=115 \mathrm{pH}$ is the inductance of the front-end SQUID, and $\hbar=h / 2 \pi$ is the Planck constant.

We have performed a calibration of the sensors following the procedure developed by D. D. Awschalom et al. ${ }^{15}$ Small Pb filings were obtained from $99.99 \% \mathrm{~Pb}$ foil (Goodfellow). By melting these filings under Ar atmosphere, we got small $\mathrm{Pb}$ spheres, which are stabilized by their surface tension. $\mathrm{A} \mathrm{Pb}$ sphere with radius $R=45 \mu \mathrm{m}$ was used as the sample for the calibration of our microsusceptometer. As the device is cooled through the transition temperature of $\mathrm{Pb}$ $\left(T_{c} \approx 7 \mathrm{~K}\right)$, we measure a relative inductance variation of $\Delta M / M=3 \times 10^{-3}$, consistent with the geometrical estimations done by M. B. Ketchen et al. ${ }^{16}$ The calibration factor can then be obtained from the formula

$$
F=\frac{4 H \pi R^{3}}{3(1+D \chi) \delta \phi}=10^{-11} \mathrm{Am}^{2} / \phi_{0} .
$$

Here, $H=4.8 \mathrm{~A} / \mathrm{m}$ is the estimated magnetic field at the sample's position, $D=1 / 3$ is the demagnetization factor of a sphere, $\chi=-1$, and $\delta \phi$ is the variation of the flux in the SQUID caused by the superconducting transition of the $\mathrm{Pb}$ sphere. Taking into account the measured flux noise of these sensors, we can estimate their spin sensitivity, that is, the figure of merit proposed by Ketchen et al. ${ }^{17}$ Our spin sensitivity (each spin having a moment of $\mu_{\mathrm{B}}$, where $\mu_{\mathrm{B}}$ is the Bohr magneton) is $\sim 9.6 \times 10^{5} \mu_{\mathrm{B}} / \mathrm{Hz}^{1 / 2}$ at $4.2 \mathrm{~K}$ and $\sim 2.1 \times 10^{5} \mu_{\mathrm{B}} / \mathrm{Hz}^{1 / 2}$ at $13 \mathrm{mK}$ for a sensing area of approximately $350 \times 600 \mu \mathrm{m}^{2}$. We have also fabricated SQUIDsusceptometers with no intermediate loop, for which we measured a calibration factor $1.15 \times 10^{-12} \mathrm{~A} \mathrm{~m}^{2} / \phi_{0}$ and a spin sensitivity of $\sim 2.4 \times 10^{4} \mu_{\mathrm{B}} / \mathrm{Hz}^{1 / 2}$ at $13 \mathrm{mK}$ for a sensing area of approximately $50 \times 200 \mu \mathrm{m}^{2}$. This follows the known rule that better sensitivities are achieved with smaller dimensions. ${ }^{17}$ Previous results have indeed shown sensitivities of $10^{3}$ spins $/ \mathrm{Hz}^{1 / 2}$ at $290 \mathrm{mK}$ for a sensing area of 25 $\times 25 \mu \mathrm{m}^{2},{ }^{18} 2 \times 10^{2}$ spins $/ \mathrm{Hz}^{1 / 2}$ at $200 \mathrm{mK}$ for a circular sensing area with $4 \mu \mathrm{m}$ diameter, ${ }^{19}$ and $10^{2}$ spins $/ \mathrm{Hz}^{1 / 2}$ at $4.2 \mathrm{~K}$ for a sensing area of $200 \times 200 \mathrm{~nm}^{2}{ }^{20}$

Figure 4 illustrates the extreme performance of our integrated susceptometers. The top panel shows susceptibility data measured at $4.2 \mathrm{~K}$ on a micron-sized crystal of $\mathrm{Mn}_{12}-\mathrm{Bz}$ single-molecule magnets ${ }^{21}$ placed directly on the SQUID loop. In a single run, the frequency dependent susceptibility enables measuring, under identical conditions, the 


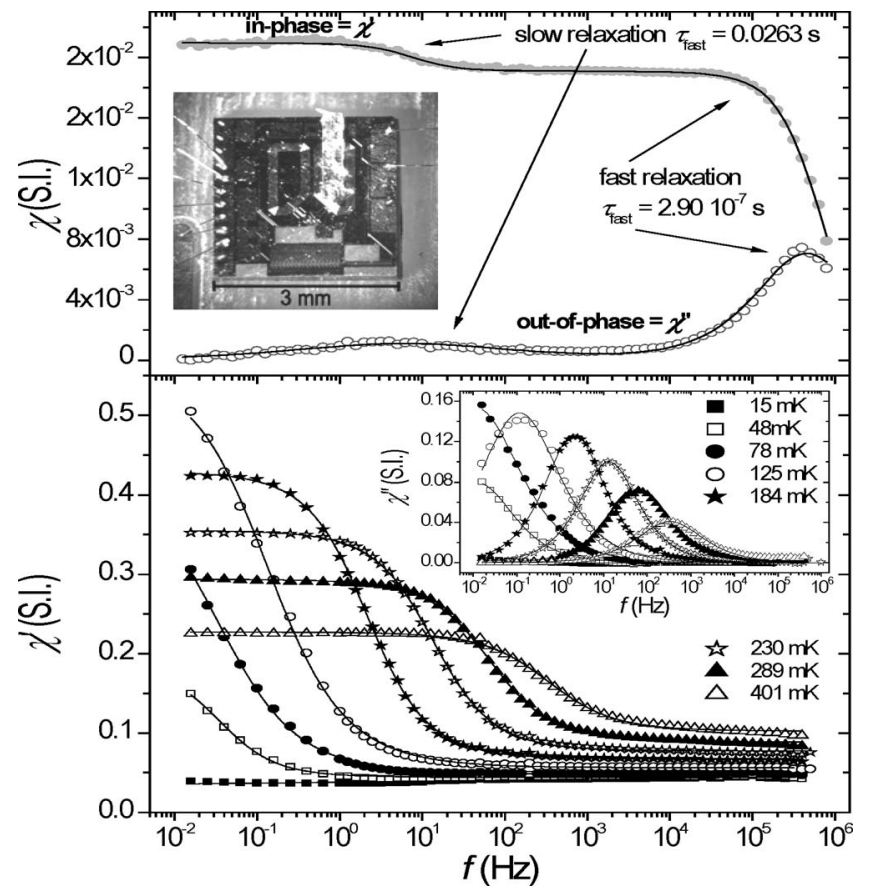

FIG. 4. (Top) In-phase and out-of-phase ac susceptibility components of a $100 \times 50 \times 20 \mu \mathrm{m}^{3}$ single crystal of $\mathrm{Mn}_{12} \mathrm{Bz}$ single-molecule magnets (SSM) at $T=4.2 \mathrm{~K}$. Measurements within the whole bandwidth of the system enable us to observe the magnetic relaxation of the two molecular species present in this sample. The respective relaxation times have been determined by fits of a combination of two Cole-Cole laws to the experimental data (solid lines). The inset shows a larger $800 \times 200 \times 200 \mu \mathrm{m}^{3}$ single crystal of $\mathrm{HoW}_{10}$ SSM attached with vacuum grease onto the intermediate loop (pick-up coil) of a SQUID susceptometer. (Bottom) Real and imaginary component of the susceptibility of the same $\mathrm{HoW}_{10}$ crystal fitted to Cole-Cole functions (solid lines). Measurements at different temperatures down to $15 \mathrm{mK}$ provide much information about the nature of the relaxation process taking place in the sample.

very different magnetic relaxation times $\tau$ of the two species of $\mathrm{Mn}_{12}$ clusters present in this sample: the "fast relaxing species," about $90 \%$ of the clusters in this compound, with relaxation times of order $3 \times 10^{-7} \mathrm{~s}$ at $4.2 \mathrm{~K}$, and the remaining $10 \%$ of standard, or "slow relaxing," $\mathrm{Mn}_{12}$ molecules, for which $\tau \approx 0.026 \mathrm{~s}$. The bottom panel shows measurements performed at very low temperatures, down to $15 \mathrm{mK}$, on $\mathrm{HoW}_{10}$ mononuclear single-molecule magnets. The relaxation rates of these nanomagnets remain sufficiently large even down to the close vicinity of the absolute zero, which our powerful dilution refrigerator enables us to explore.

The susceptometry setup developed by us combines the high sensitivity and broad bandwidth of the Magnicon/PTB SQUIDs sensors with the possibility of measuring samples in direct contact with the ${ }^{3} \mathrm{He}-{ }^{4} \mathrm{He}$ mixture of a dilution refrigerator unit. The device, fabricated by a simple modification of the electrical circuit carried out in our laboratories can be applied to investigate quantum dynamics of single-molecule magnets and even isolated paramagnetic spins in the neighborhood of the absolute zero of temperature. Samples of micrometer size can be easily placed onto the pick-up coils, gluing them with vacuum grease. Thanks to their high sensitivity, these susceptometers should also enable the detection of single layers of molecular nanomagnets, therefore providing a relatively simple tool to ascertain if these mate- rials preserve their interesting magnetic properties (magnetic memory and quantum tunnelling). For this, we plan to deposit dots of a controlled amount of sample at a desired location by using dip-pen lithography. Preliminary results have shown that this technique enables depositing ferritin proteins with single-molecule precision. ${ }^{22}$

We thank K. Awaga and K. Takeda for letting us use the $\mathrm{Mn}_{12}-\mathrm{Bz}$ crystals and E. Coronado and S. Cardona for the synthesis of the $\mathrm{HoW}_{10}$ compound. Funding from DGA (Grant No. PI091/08 NABISUP), MOLBIT (Grant No. MAT2006-13765-C02), MICINN (Grant No. MAT200913977 MOLCHIP), and Integrated Action (Grant No. HA2006-0051) with Germany and "Molecular Nanoscience" from the Consolider 2010 Programme is acknowledged.

${ }^{1}$ J. R. Friedman, M. P. Sarachik, J. Tejada, and R. Ziolo, Phys. Rev. Lett. 76, 3830 (1996); J. M. Hernández, X. X. Zhang, F. Luis, J. Bartolomé, J. Tejada, and R. Ziolo, Europhys. Lett. 35, 301 (1996); L. Thomas, F. Lionti, R. Ballou, D. Gatteschi, R. Sessoli, and B. Barbara, Nature (London) 383, 145 (1996).

${ }^{2}$ D. D. Awschalom, J. F. Smyth, G. Grinstein, D. P. DiVincenzo, and D. Loss, Phys. Rev. Lett. 68, 3092 (1992).

${ }^{3}$ F. Luis, F. L. Mettes, J. Tejada, D. Gatteschi, and L. J. de Jongh, Phys. Rev. Lett. 85, 4377 (2000).

${ }^{4}$ S. Ghosh, R. Parthasarathy, T. F. Rosenbaum, and G. Aeppli, Science 296, 2195 (2002)

${ }^{5}$ S. Bertaina, S. Gambarelli, T. Mitra, B. Tsukerblat, A. Müller, and B. Barbara, Nature (London) 453, 203 (2008).

${ }^{6}$ S. Ghosh, T. F. Rosenbaum, G. Aeppli, and S. N. Coppersmith, Nature (London) 425, 48 (2003).

7. A. Timco, S. Carretta, F. Troiani, F. Tuna, R. J. Pritchard, C. A. Muryn, E. J. L. McInnes, A. Ghirri, A. Candini, P. Santini, G. Amoretti, M. Affronte, and R. E. P. Winpenny, Nat. Nanotechnol. 4, 173 (2009).

${ }^{8}$ J. Tejada, E. M. Chudnovsky, E. M. del Barco, J. M. Hernández, and T. P. Spiller, Nanotechnology 12, 181 (2001).

${ }^{9}$ C. P. Foley and H. Hilgekamp, Supercond. Sci. Technol. 22, 064001 (2009).

${ }^{10}$ S. T. P. Boyd, V. Kotsubo, R. Cantor, A. Theodorou, and J. A. Hall, IEEE Trans. Appl. Supercond. 19, 697 (2009).

${ }^{11}$ D. Drung, C. Aßmann, J. Beyer, A. Kirste, M. Peters, F. Ruede, and T. Schurig, IEEE Trans. Appl. Supercond. 17, 699 (2007).

${ }^{12}$ E. S. Sadki, S. Ooi, and K. Hirata, Appl. Phys. Lett. 85, 6206 (2004).

${ }^{13}$ M. J. Martínez-Pérez, J. Sesé, R. Córdoba, F. Luis, D. Drung, and T. Schurig, Supercond. Sci. Technol. 22, 125020 (2009).

${ }^{14}$ I. Guillamón, H. Suderow, S. Vieira, A. Fernandez-Pacheco, J. Sesé, R. Córdoba, J. M. De Teresa, and M. R. Ibarra, New J. Phys. 10, 093005 (2008).; I. Guillamón, H. Suderow, A. Fernández-Pacheco, J. Sesé, R. Córdoba, J. M. De Teresa, M. R. Ibarra, and S.Vieira, Nature Phys. 5, 651 (2009).

${ }^{15}$ D. D. Awschalom, J. R. Rozen, M. B. Ketchen, W. J. Gallaguer, A. W. Kleinsasser, R. L. Sandstrom, and B. Bumble, Appl. Phys. Lett. 53, 2108 (1988).

${ }^{16}$ M. B. Ketchen, T. Kopley, and H. Ling, Appl. Phys. Lett. 44, 1008 (1984).

${ }^{17}$ M. B. Ketchen, D. D. Awschalom, W. J. Gallaguer, A. W. Kleinsasser, R. L. Sandstrom, J. R. Rozen, and B. Bumble, IEEE Trans. Magn. 25, 1212 (1989).

${ }^{18}$ F. Wellstood, C. Urbina, and J. Clarke, Appl. Phys. Lett. 54, 2599 (1989).

${ }^{19}$ M. E. Huber, N. C. Koshnick, H. Bluhm, L. J. Archuleta, T. Azua, P. G. Björnsson, B. W. Gardner, S. T. Halloran, E. A. Lucero, and K. A. Moler, Rev. Sci. Instrum. 79, 053704 (2008).

${ }^{20}$ C. Granata, E. Esposito, A. Vettoliere, L. Petti, and M. Russo, Nanotechnology 19, 275501 (2008)

${ }^{21}$ K. Takeda, K. Awaga, T. Inabe, A. Yamaguchi, H. Ishimoto, T. Tomita, H. Mitamura, T. Goto, N. Mori, and H. Nojiri, Phys. Rev. B 65, 094424 (2002).

${ }^{22}$ E. Bellido, R. de Miguel, D. Ruiz-Molina, A. Lostao and D. Maspoch, Adv. Mater. 22, 352 (2009). 